\title{
Real-World Safety and Effectiveness of Dimethyl Fumarate in Hispanic or Latino Patients with Multiple Sclerosis: 3-Year Results from ESTEEM
}

\author{
Angel Chinea - Lilyana Amezcua - Wendy Vargas · Annette Okai • \\ Mitzi J. Williams · Ray Su • Becky Parks · Jason P. Mendoza • \\ James B. Lewin · Cynthia C. Jones
}

Received: April 2, 2020 / Published online: May 29, 2020

(C) The Author(s) 2020

\section{ABSTRACT}

Introduction: Compared with the non-Hispanic/non-Latino population, Hispanic/Latino patients with multiple sclerosis (MS) are reported to exhibit greater disease severity. Geographical location and genetics play a role in differences observed across Hispanic/Latino

Digital features To view digital features for this article go to https://doi.org/10.6084/m9.figshare.12205952.

B. Parks was an employee of Biogen at the time the research was conducted.

\section{A. Chinea}

From the San Juan Multiple Sclerosis Center, Guaynabo, Puerto Rico

\section{Amezcua}

Keck School of Medicine, University of Southern California, Los Angeles, CA, USA

\section{W. Vargas}

Columbia University Medical Center, New York, NY, USA

A. Okai

Multiple Sclerosis Treatment Center of Dallas, Dallas, TX, USA

\section{J. Williams}

Joi Life Wellness MS Center, Atlanta, GA, USA

R. Su $\cdot$ B. Parks $\cdot$ J. P. Mendoza $\cdot$ J. B. Lewin

C. C. Jones $(\bowtie)$

Biogen, Cambridge, MA, USA

e-mail: cynthia.jones@biogen.com subpopulations. We evaluated real-world safety and effectiveness of dimethyl fumarate (DMF) on MS disease activity in Hispanic/Latino patients.

Methods: ESTEEM is an ongoing, 5-year, multinational, prospective study evaluating long-term safety and effectiveness of DMF in patients with MS. This interim analysis included patients newly prescribed DMF in routine practice at 394 sites globally.

Results: Overall, 4986 non-Hispanic/nonLatino and 98 Hispanic/Latino patients were analyzed; median (range) follow-up was 18 (2-37) months. Unadjusted annualized relapse rates (ARRs) for 12 months before DMF initiation versus 36 months post DMF initiation, respectively, were: non-Hispanic/non-Latino patients, $0.82(95 \%$ CI $0.80-0.84)$ versus 0.10 (95\% CI 0.09-0.10), 88\% lower ARR $(P<0.0001) ; \quad$ Hispanic/Latino patients, 0.80 $(95 \%$ CI $0.65-1.00)$ versus $0.09 \quad(95 \%$ CI $0.06-0.14), 89 \%$ lower ARR $(P<0.0001)$. In total, 28 (29\%) Hispanic/Latino patients reported adverse events leading to treatment discontinuation; gastrointestinal (GI) disorders ( $n=10,10 \%)$ were the most common, consistent with the non-Hispanic/non-Latino population (8\%). Median lymphocyte counts decreased by approximately $24 \%$ in the first year (vs 36\% decrease in non-Hispanic/nonLatino patients) then remained stable and above the lower limit of normal in most patients. 
Conclusions: Relapse rates remained low in Hispanic/Latino patients, consistent with nonHispanic/non-Latino patients. The safety profile of DMF in Hispanic/Latino patients was consistent with safety findings from the non-Hispanic/non-Latino ESTEEM population, demonstrating the real-world treatment benefit of DMF in the Hispanic/Latino patient cohort.

Keywords: Dimethyl fumarate; Multiple sclerosis; Safety and effectiveness

\section{Key Summary Points}

Why carry out this study?

Delayed-release dimethyl fumarate (DMF) has demonstrated clinically meaningful, sustained efficacy and a favorable benefit-risk profile in patients with relapsing forms of multiple sclerosis (MS)

ESTEEM is an ongoing, 5-year, multinational, prospective study evaluating long-term safety and effectiveness of DMF newly prescribed to patients with MS

Geographical location and genetics play a role in differences observed across Hispanic subpopulations

In this interim analysis of ESTEEM, we evaluated real-world safety and effectiveness of DMF in the largest subgroup of DMF-treated Hispanic patients studied to date

\section{What was learned from the study?}

The safety profile and the effectiveness of DMF on relapse rates in Hispanic patients were consistent with findings observed from the non-Hispanic population

These analyses demonstrate the realworld treatment benefit of DMF in Hispanic patients, consistent with findings in the overall ESTEEM population

\section{INTRODUCTION}

Ethnic minority populations continue to be underrepresented in multiple sclerosis (MS) research in the real-world setting and in clinical trials. For example, of approximately 60,000 published articles on MS found through a PubMed literature review conducted in 2014 by Khan and colleagues [1], only 23 focused on Hispanic-American patient populations with MS. The underrepresentation of these populations leads to limited data on the effectiveness of treatments in these groups of patients and underscores the lack of an evidence-based approach used in treatment. Also, the limited studies and subgroup analyses that have been conducted have labeled these groups differently, as the nomenclature in the literature is inconsistent.

Dimethyl fumarate (DMF) and diroximel fumarate are oral, disease-modifying therapies approved for treating relapsing forms of MS. DMF and diroximel fumarate rapidly undergo esterase cleavage to monomethyl fumarate, a pharmacologically active metabolite $[2,3]$. As of January 31,2020 , more than 445,000 patients have been treated with DMF worldwide, representing more than 875,000 patient-years of exposure. Of these, 6335 patients $(14,241$ patient-years) were from clinical trials. DMF has demonstrated strong, sustained efficacy and a favorable benefit-risk profile in patients with relapsing-remitting multiple sclerosis in the pivotal phase 3 studies DEFINE and CONFIRM, the ongoing open-label ENDORSE extension study, and real-world studies [4-6]. Although data from DEFINE and CONFIRM demonstrated a significant reduction in relapses in Hispanic patients, the sample size $(n=54)$ was too small to draw definitive conclusions regarding the effects of DMF in this subgroup [7]. ESTEEM (ClinicalTrials.gov identifier NCT02047097) is an ongoing, 5-year, multinational, prospective, noninterventional, phase 4 study evaluating the long-term safety and effectiveness of DMF in patients treated in real-world clinical practice. In this interim analysis, we evaluated the safety and effectiveness of DMF on MS disease activity in non-Hispanic/non-Latino patients 
and Hispanic/Latino patients, and in a cohort of Hispanic/Latino patients who switched from another therapy to DMF (received prior interferon [IFN] or glatiramer acetate [GA] at any time following diagnosis of MS).

\section{METHODS}

\section{Study Design and Patients}

This interim analysis of the ESTEEM study data (data cutoff April 3, 2019) included patients with MS who had been newly prescribed DMF in routine practice at 394 sites globally. Hispanic/Latino patients were those who self-reported their ethnicity as "Hispanic or Latino". Non-Hispanic/non-Latino patients were defined as patients who did not select "Hispanic or Latino" as their ethnicity. Of note, race was captured separately and was also self-reported; among the subgroup of patients who identified their ethnicity as Hispanic or Latino, most identified their race as "White".

Patients under the age of 12 years are excluded from the ESTEEM study given the challenges of enrolling a sufficient number of patients, as less than $1 \%$ of the MS population are 11 years of age or under [8]. Patients aged between 12 and less than 18 years are also excluded where enrollment of pediatric patients is prohibited. In all, 5000 patients will be monitored for 5 years via routine clinical visits. The primary objective of the ESTEEM study was to determine the incidence, type, and pattern of serious adverse events (SAEs), including serious infections, and of AEs leading to treatment discontinuation. Magnetic resonance imaging data that would enable the characterization of disease severity at baseline were unavailable. A secondary objective of the interim analysis reported here was to consider the effectiveness of DMF on MS disease activity in non-Hispanic/ non-Latino patients, Hispanic/Latino patients, and a subgroup of Hispanic/Latino patients who switched from another therapy to DMF (received prior IFN/GA at any time from diagnosis). This was achieved through the assessment of annualized relapse rate (ARR), the proportion of patients with a relapse, and the distribution of the number of relapses. The incidence of treatment discontinuation was also assessed.

The study was conducted in accordance with relevant US federal regulations, the Declaration of Helsinki, and the International Council on Harmonisation Guideline for Good Clinical Practice. Approvals were granted by relevant institutional ethics committees for study protocol and amendments, and written assent and consent forms were obtained from each patient and his or her parent or legal guardian. ESTEEM was registered at ClinicalTrials.gov (NCT02047097).

\section{Statistical Analysis}

ARRs were obtained by fitting a repeated-measure negative binomial model (ARR was defined as the total number of relapses during the study for all patients divided by the total number of patient-years in the study adjusted for appropriate prognostic factors, and time-to-event endpoints analyzed using Kaplan-Meier estimates). Descriptive summary statistics were calculated.

\section{RESULTS}

\section{Study Population}

In total, 4986 non-Hispanic/non-Latino and 98 Hispanic/Latino patients, including 64 Hispanic/Latino patients who switched to DMF from IFN/GA, received at least one dose of DMF, and were included in this analysis. Baseline characteristics are shown in Table 1; the majority of non-Hispanic/non-Latino patients were of European background (56\%), whereas 98\% of Hispanic/Latino patients were from the USA (including Puerto Rico). The mean (SD) age of Hispanic/Latino patients was 40 (11.6) years, and $79 \%$ of these patients were female. No patients in the Hispanic/Latino patient group were under 18 years of age (non-Hispanic/nonLatino population, $n=10$; Hispanic/Latino population, $n=0$ [minimum age, 19 years]). Although the percentage of patients over 40 years of age was greater in the non-Hispanic/ 
Table 1 Patient baseline characteristics

\section{$n$ (\%), unless otherwise specified}

Sex, F

Region

USA, including Puerto Rico

Western Europe, Canada, New Zealand, and Australia

Eastern Europe plus India, Mexico, and Argentina

Median (range) age at MS diagnosis, years

Median (range) duration between most recent relapse and enrollment, months

Median (range) number of relapses within past 12 months

Mean (SD) DMF treatment duration, months

Patients with minimum 1-year follow-up

Patients with minimum 2-year follow-up

Median (range) duration in ESTEEM, months

Mean (SD) EDSS score ${ }^{\mathrm{b}}$

Prior MS treatment

Glatiramer acetate

Intramuscular IFN $\beta$-1a

Subcutaneous IFN $\beta$-1a

IFN $\beta-1 b$

Fingolimod

Teriflunomide

Tobacco use

Current user

Non-tobacco user
Non-Hispanic/Non-Latino
patients

$n=4986$

$40(11.2)$

$2520(51)$

$51(52)$

2465 (49)

$47(48)$

$3681(74)$

$77(79)$

1301 (26)

$96(98)$

$2811(56)$

1 (1)

874 (18)

1 (1)

$34(9-83)$

$34(17-67)$

$5.8(0-325)$

$6.8(0-410)$

$1(0-6)$

$1(0-4)$

$21.5(16.3)$

$19.0(15.0)$

$3179(64)$

$59(60)$

$1915(38)$

$32(33)$

$24.4(0-63)$

$23.8(1-55)$

$2.10(1.48)$

2.46 (2.35)

3181 (64)

67 (68)

1255 (40)

30 (45)

958 (30)

28 (42)

1511 (48)

33 (49)

$191(6)$

4 (6)

284 (9)

9 (13)

218 (7)

3 (5)

2803

68

719 (26)

$2084(74)$

$61(90)$

$D M F$ dimethyl fumarate, EDSS Expanded Disability Status Scale, IFN interferon, $M S$ multiple sclerosis

a Age $=$ (year diagnosed or year of first MS symptom - year of birth +1 )

b For patients with available data from EDSS assessment at enrollment. Non-Hispanic/Non-Latino patients, $n=2798$; Hispanic/Latino patients, $n=23$ 
non-Latino cohort $(49 \% ; 2465 / 4986)$ than the Hispanic/Latino population $(48 \% ; 47 / 98)$, this difference was not of statistical significance.

\section{ARR and Relapses}

The mean (SD) duration between most recent relapse and enrollment was 21.0 (35.54) months for the non-Hispanic/non-Latino population and 31.8 (63.92) months for the Hispanic/ Latino population. The mean (SD) number of relapses in the prior 3 years was 1.4 (1.32) for the non-Hispanic/non-Latino population and 1.4 (2.06) for the Hispanic/Latino population, indicating no difference between the two groups. Among non-Hispanic/non-Latino patients, ARR 12 months before DMF initiation was $0.82 \quad(95 \%$ CI $0.80-0.84)$ and after 36 months it was 0.10 (95\% CI 0.09-0.10; $P<0.0001 ;$ Fig. 1). Among Hispanic/Latino patients, ARR 12 months before DMF initiation was $0.80 \quad(95 \% \quad \mathrm{CI} \quad 0.65-1.00)$ and after 36 months it was 0.09 (95\% CI $0.06-0.14$; $P<0.0001$; Fig. 1).

For the Hispanic/Latino IFN/GA switch population, ARR 12 months before switching was 0.73 (95\% CI $0.54-1.00)$ versus 0.10 (95\% CI $0.05-0.20)$ after 36 months $(P<0.0001$, Fig. 1$)$. The estimated proportion of relapse-free patients at 36 months was similar (80-88\%) in non-Hispanic/non-Latino patients, Hispanic/ Latino patients, and Hispanic/Latino patients who switched therapy (Table 2).

\section{Safety and Discontinuations}

A total of 937 (19\%) non-Hispanic/non-Latino patients and 28 (29\%) Hispanic/Latino patients reported any $\mathrm{AE}$ leading to treatment discontinuation (Table 3). Gastrointestinal (GI) disorders were the most commonly cited (nonHispanic/non-Latino patients, $n=385$ [8\%];

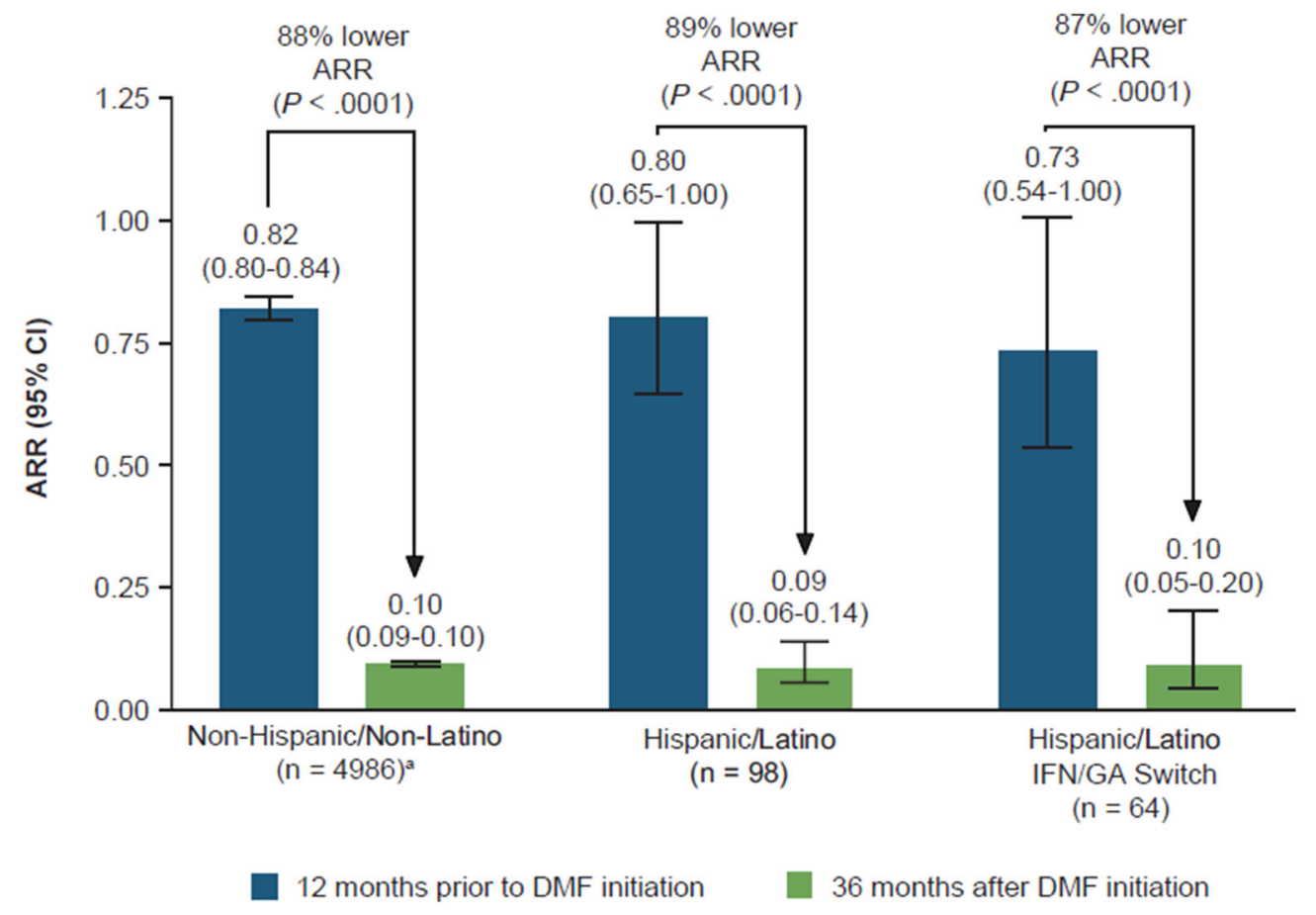

Fig. 1 ARR in the 12 months before and 36 months after DMF initiation for the non-Hispanic/non-Latino, Hispanic/Latino, and Hispanic/Latino IFN/GA switch subgroups. $A R R$ annualized relapse rate, $D M F$ dimethyl fumarate, $G A$ glatiramer acetate, $I F N$ interferon. ${ }^{2}$ Patients with missing relapse-rate data 12 months before DMF initiation were excluded from the ARR analysis. ARRs were obtained by fitting a repeated-measure negative binomial model 
Table 2 Proportion of relapse-free patients in the 36 months after DMF initiation

\begin{tabular}{llll}
\hline & $\begin{array}{l}\text { Non-Hispanic/Non-Latino } \\
\text { patients } \\
\boldsymbol{n}=\mathbf{4 9 8 6}\end{array}$ & $\begin{array}{l}\text { Hispanic/Latino } \\
\text { patients } \\
\boldsymbol{n}=\mathbf{9 8}\end{array}$ & $\begin{array}{l}\text { Hispanic/Latino IFN/GA } \\
\text { switch patients } \\
\boldsymbol{n}=\mathbf{6 4}\end{array}$ \\
\hline $\begin{array}{l}\text { Proportion of patients without }_{\text {MS relapse }}^{\mathrm{a}} \\
\text { M }\end{array}$ & 80 & 84 & 88 \\
\hline
\end{tabular}

Values are given as percentage

$D M F$ dimethyl fumarate, $G A$ glatiramer acetate, $I F N$ interferon, $M S$ multiple sclerosis

a Kaplan-Meier analyses

Hispanic/Latino patients, $n=10[10 \%])$, the majority of these GI-related discontinuations occurring within the first month of DMF treatment (non-Hispanic/non-Latino population, $n=271 / 385$ [70\%]; Hispanic/Latino, $n=8 / 10$
[80\%]). SAEs were reported in 239/4986 (5\%) non-Hispanic/non-Latino patients and 6/98 (6\%) Hispanic/Latino patients.

Table 3 Most common AEs leading to DMF discontinuation in the overall ESTEEM non-Hispanic/non-Latino and Hispanic/Latino patient population

\begin{tabular}{|c|c|c|}
\hline Category, $n(\%)^{\mathrm{a}}$ & $\begin{array}{l}\text { Non-Hispanic/Non-Latino patients } \\
n=4986\end{array}$ & $\begin{array}{l}\text { Hispanic/Latino patients } \\
n=98\end{array}$ \\
\hline Any AE leading to treatment discontinuation & $937(19)$ & $28(29)$ \\
\hline GI disorders & $385(8)$ & $10(10)$ \\
\hline Diarrhea & $115(2)$ & $3(3)$ \\
\hline Nausea & $105(2)$ & $2(2)$ \\
\hline Abdominal pain & $67(1)$ & $2(2)$ \\
\hline Vomiting & $63(1)$ & $1(1)$ \\
\hline Skin and subcutaneous tissue disorders & $103(2)$ & $7(7)$ \\
\hline Rash & $18(<1)$ & $2(2)$ \\
\hline Rash, generalized & $5(<1)$ & $2(2)$ \\
\hline Infections & $30(<1)$ & $3(3)$ \\
\hline Investigations & $93(2)$ & $2(2)$ \\
\hline Lymphocyte count decreased & $52(1)$ & $2(2)$ \\
\hline Nervous system disorders & $100(2)$ & $3(3)$ \\
\hline Blood and lymphatic system disorders & $142(3)$ & $3(3)$ \\
\hline General disorders and administration site conditions & $67(1)$ & $2(2)$ \\
\hline
\end{tabular}

Values are given as number (percentage)

$A E$ adverse event, $D M F$ dimethyl fumarate, $G I$ gastrointestinal

${ }^{a}$ AEs occurring in $>1$ patient in the Hispanic/Latino subgroup. AEs were coded using the Medical Dictionary for Regulatory Activities (MedDRA) System Organ Class (SOC) and Preferred Term (PT). Percentages were calculated on the basis of total number of patients in each group. A patient was counted only once in each MedDRA SOC or PT 


\section{Absolute Lymphocyte Counts}

Mean lymphocyte change over time for a duration of 36 months is shown in Fig. 2. In Hispanic/Latino patients, among patients with available lymphocyte data $(n=31)$, median absolute lymphocyte count (ALC) decreased $24 \%$ from baseline $\left(1.96 \times 10^{9} / \mathrm{L}\right)$ to month 12 $\left(1.41 \times 10^{9} / \mathrm{L}\right)$ and then remained stable. Median lymphocyte count remained above the lower limit of normal $\left(0.91 \times 10^{9} / \mathrm{L}\right)$; however, the number of patients with median lymphocyte count by month 36 was limited $(n=9)$. In all other patients with available lymphocyte data $(n=2380)$, median ALC decreased 36\% from baseline $\left(1.97 \times 10^{9} / \mathrm{L}\right)$ to month 12 $\left(1.22 \times 10^{9} / \mathrm{L}\right)$ and then remained stable.

\section{DISCUSSION}

We report real-world safety and effectiveness of DMF on MS disease activity in non-Hispanic/ non-Latino and Hispanic/Latino patients, including those Hispanic/Latino patients who also switched therapy to DMF from prior IFN/ GA. Compared with the 12 months before DMF initiation, ARR was significantly lower in the 36 months after DMF initiation in the overall
Hispanic/Latino cohort, and in Hispanic/Latino patients who switched from prior IFN/GA. These results are consistent with ARRs reported in the non-Hispanic/non-Latino population. No unexpected safety findings were observed in the Hispanic/Latino population, with $\mathrm{AE}$ and SAE profiles similar to those seen in non-Hispanic/ non-Latino patients. Less than one-third of Hispanic/Latino patients reported AEs leading to treatment discontinuation, with GI disorders being the most commonly reported; this is very similar to reports from the non-Hispanic/nonLatino population (8\%). It is not possible to draw a conclusion about AEs leading to treatment discontinuation owing to the small sample size; however, these results warrant further investigation with continued follow-up or additional studies.

The majority (80\%) of GI disorders for the Hispanic/Latino population occurred within the first month of treatment. In this study, we observed a median $24 \%$ reduction in absolute lymphocyte count over the first year of DMF treatment in Hispanic/Latino patients, and absolute lymphocyte count remained above the lower limit of normal in all Hispanic/Latino patients, even at month $36(n=9)$. Tolerability and effectiveness of treatment with DMF across

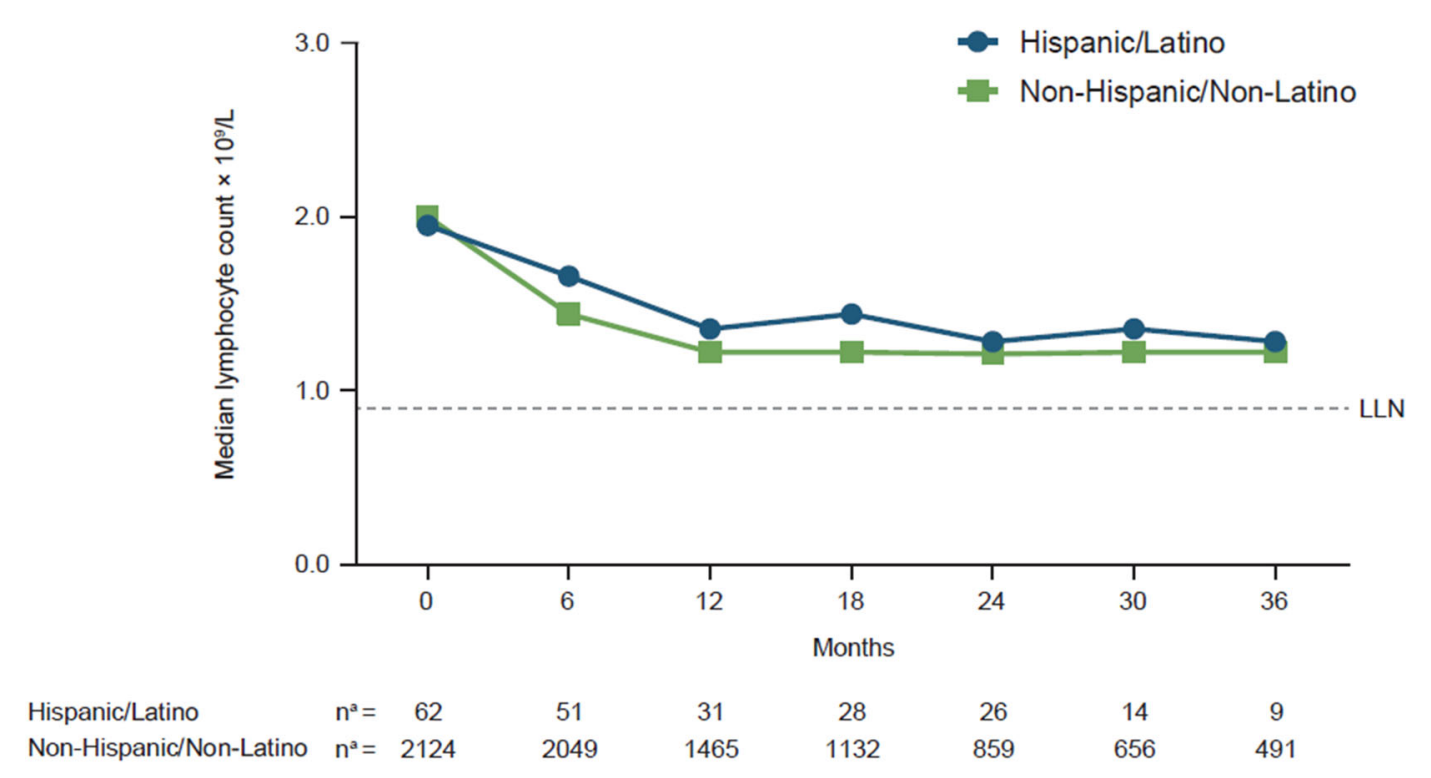

Fig. 2 Median absolute lymphocyte count over time in Hispanic/Latino patients. $L L N$ lower limit of normal $\left(0.91 \times 10^{9} / \mathrm{L}\right)$. ${ }^{a} n$ number of patients with available data from absolute lymphocyte count assessment at the specified time point 
different racial groups may vary because clinically important differences in the course of MS and response to therapies exist among patients of different racial and ethnic backgrounds [9-13]. For example, Hispanic-American patients with MS may be more likely to experience optic neuritis, transverse myelitis, and low levels of vitamin $\mathrm{D}$-conditions that can be considered risk factors for MS [14, 15]. These patients also tend to be of a younger age at disease onset and have a more aggressive disease course with more rapid disability progression compared with white patients with MS [9]. Our study provides evidence that, in this small cohort of Hispanic/Latino patients, DMF is tolerated and effective in routine clinical practice.

One of the primary strengths of the ESTEEM study is its design, which enables prospective observation of patients in routine clinical practice over time. In this interim analysis, DMF outcomes were analyzed in the largest Hispanic/ Latino cohort used to date. We have reported on outcomes over the longest follow-up duration compared with previous studies. Potential differences in disease pathophysiology among ethnic groups merit further study and may have implications for the treatment of patients with MS. To enable more comprehensive and accurate data to be obtained about MS and its treatment in underserved populations, it is important that health care providers discuss participation in clinical trials with all patients, with the aim of increasing patient participation and subsequently the generalizability of clinical trial findings.

Study limitations include the observational open-label design, the lack of magnetic resonance imaging baseline data to accurately characterize the disease severity of this population, and the short follow-up duration. Nevertheless, this prospective study provides preliminary data that suggest that DMF outcomes in this Hispanic/Latino cohort are similar to those that have been observed in the nonHispanic/non-Latino population, and could be as effective.

Another limitation of this study is that most of the patients in this subgroup were from the USA and therefore may not be representative of a global MS population. Stratified analyses based on ethnicity are not available for patients from Europe because of differences in data collection and data privacy regulations.

In conclusion, these safety and effectiveness analyses demonstrate the real-world treatment benefit of DMF in Hispanic/Latino patients, consistent with findings in the overall ESTEEM population. Similar findings also were observed in a subgroup analysis of African-American patients [16]. The ESTEEM study is ongoing; therefore, further insights into the safety and effectiveness of DMF in different ethnic groups may be obtained from the final 5-year study results.

\section{ACKNOWLEDGEMENTS}

Funding/Support. This study, including the Rapid Service Fee, was sponsored by Biogen (Cambridge, MA, USA).

Medical Writing and/or Editorial Assistance. Biogen provided funding for medical writing support in the development of this paper; Chris Traylen, $\mathrm{PhD}$, and Katherine Ayling-Rouse, MSc, from Excel Scientific Solutions wrote the first draft of the manuscript based on input from authors, and Adrienne Trout, BSc, from Excel Scientific Solutions copyedited and styled the manuscript per journal requirements. Biogen reviewed and provided feedback on the paper to the authors. The authors had full editorial control of the paper and provided their final approval of all content.

Authorship. All named authors meet the International Committee of Medical Journal Editors (ICMJE) criteria for authorship for this article, take responsibility for the integrity of the work as a whole, and have given their approval for this version to be published.

Disclosures. Dr. Chinea has received advisory/consulting fees from Biogen, Genentech, Novartis, Sanofi Genzyme, and Teva Neuroscience; research support from Biogen, Novartis, and Sanofi Genzyme; and speaker fees from Biogen, Genentech, Novartis, Sanofi Genzyme, 
and Teva. Dr. Amezcua has received advisory/consulting fees from Novartis and Genzyme, and research support from Biogen and MedDay. Dr. Vargas has received advisory/consulting fees from Alexion, Biogen, Genentech, and Octapharma, and research support from Teva. Dr. Okai has received advisory/consulting fees from Biogen, Celgene, EMD Serono, Genentech, Genzyme, and TG Therapeutics; support from Biogen, Novartis, Sanofi Genzyme, and TG Therapeutics; and speaker fees from Biogen, EMD Serono, Genentech, Novartis, Sanofi Genzyme, and Teva. Dr. Williams has received advisory/consulting fees from Biogen, Celgene, EMD Serono, Genentech, Novartis, Sanofi Genzyme, and Teva Neuroscience, and speaker fees from Biogen, EMD Serono, Genentech, Novartis, Sanofi Genzyme, and Teva. Drs. $\mathrm{Su}, \mathrm{Mendoza}$, Lewin, and Jones are employees of and hold stock/stock options in Biogen. B. Parks was an employee of Biogen at the time the research was conducted. Dr Parks is now employed at Blueprint Medicines, Cambridge, MA, USA.

Compliance with Ethics Guidelines. The study was conducted in accordance with relevant US federal regulations, the Declaration of Helsinki, and the International Council on Harmonisation Guideline for Good Clinical Practice. Approvals were granted by relevant institutional ethics committees for study protocol and amendments, and written assent and consent forms were obtained from each patient and his or her parent or legal guardian. ESTEEM was registered at ClinicalTrials.gov (NCT02047097).

Data Availability. All data generated or analyzed during this interim subgroup analysis are included in this published article/as supplementary information files.

Open Access. This article is licensed under a Creative Commons Attribution-NonCommercial 4.0 International License, which permits any non-commercial use, sharing, adaptation, distribution and reproduction in any medium or format, as long as you give appropriate credit to the original author(s) and the source, provide a link to the Creative Commons licence, and indicate if changes were made. The images or other third party material in this article are included in the article's Creative Commons licence, unless indicated otherwise in a credit line to the material. If material is not included in the article's Creative Commons licence and your intended use is not permitted by statutory regulation or exceeds the permitted use, you will need to obtain permission directly from the copyright holder. To view a copy of this licence, visit http://creativecommons.org/licenses/by$\mathrm{nc} / 4.0 /$.

\section{REFERENCES}

1. Khan O, Williams MJ, Amezcua L, Javed A, Larsen KE, Smrtka JM. Multiple sclerosis in US minority populations: clinical practice insights. Neurol Clin Pract. 2015;5(2):132-42.

2. TECFIDERA. TECFIDERA ${ }^{\circledR}$ (dimethyl fumarate) delayed-release capsules, for oral use. Highlights of prescribing information. Cambridge, MA, USA: Biogen Inc.; 2017. https://www.tecfidera.com/ content/dam/commercial/tecfidera/pat/en_us/pdf/ full-prescribing-info.pdf. Accessed 15 Oct 2018.

3. US Food and Drug Administration. VUMERITY ${ }^{\mathrm{TM}}$ (diroximel fumarate) delayed-release capsules, for oral use. Highlights of prescribing information Cambridge, MA, USA: Biogen Inc.; 2019. https:// www.accessdata.fda.gov/drugsatfda_docs/label/ 2019/211855s000lbl.pdf. Accessed 10 Sept 2019.

4. Fox RJ, Miller DH, Phillips JT, et al. Placebo-controlled phase 3 study of oral BG-12 or glatiramer in multiple sclerosis. N Engl J Med. 2012;367(12): 1087-97.

5. Gold R, Arnold DL, Bar-Or A, et al. Long-term effects of delayed-release dimethyl fumarate in multiple sclerosis: interim analysis of ENDORSE, a randomized extension study. Mult Scler. 2017;23(2):253-65.

6. Gold R, Kappos L, Arnold DL, et al. Placebo-controlled phase 3 study of oral BG-12 for relapsing multiple sclerosis. N Engl J Med. 2012;367(12): 1098-107.

7. Fox RJ, Gold R, Phillips JT, Okwuokenye M, Zhang A, Marantz JL. Efficacy and tolerability of delayedrelease dimethyl fumarate in black, Hispanic, and Asian patients with relapsing-remitting multiple 
sclerosis: post hoc integrated analysis of DEFINE and CONFIRM. Neurol Ther. 2017;6(2):175-87.

8. Gadoth N. Multiple sclerosis in children. Brain Dev. 2003;25(4):229-32.

9. Zhovtis Ryerson L, Green R, Confident G, et al. Efficacy and tolerability of dimethyl fumarate in White-, African- and Hispanic-Americans with multiple sclerosis. Ther Adv Neurol Disord. 2016;9(6):454-61.

10. Chinea Martinez AR, Correale J, Coyle PK, Meng X, Tenenbaum N. Efficacy and safety of fingolimod in Hispanic patients with multiple sclerosis: pooled clinical trial analyses. Adv Ther. 2014;31(10): 1072-81.

11. Fox RJ, Gold R, Phillips JT, Okwuokenye M, Zhang A, Marantz JL. Efficacy and tolerability of delayedrelease dimethyl fumarate in Black, Hispanic, and Asian patients with relapsing-remitting multiple sclerosis: post hoc integrated analysis of DEFINE and CONFIRM. Neurol Ther. 2017;6(2):175-87.
12. Mao X, Bigham AW, Mei R, et al. A genomewide admixture mapping panel for Hispanic/Latino populations. Am J Hum Genet. 2007;80(6):1171-8.

13. Price AL, Patterson N, Yu F, et al. A genomewide admixture map for Latino populations. Am J Hum Genet. 2007;80(6):1024-36.

14. Hadjixenofontos A, Beecham AH, Manrique CP, et al. Clinical expression of multiple sclerosis in Hispanic Whites of primarily Caribbean ancestry. Neuroepidemiology. 2015;44(4):262-8.

15. Langer-Gould A, Brara SM, Beaber BE, Zhang JL. Incidence of multiple sclerosis in multiple racial and ethnic groups. Neurology. 2013;80(19):1734-9.

16. Williams M, Amezcua L, Okai A, Okuda DT, Cohan S, Su R, Parks B, Mendoza JP, Lewin JB, Jones CC. Real-world safety and effectiveness of dimethyl fumarate in Black or African American patients with multiple sclerosis: 3-year results from ESTEEM. Neurol Ther. 2020 in press. 